\title{
A Spitzer Space Telescope study of dust features in planetary nebula and HII regions
}

\author{
Jeronimo Bernard-Salas ${ }^{1}$, Els Peeters ${ }^{2,3}$, Vianney Lebouteiller ${ }^{1}$ \\ Gregory C. Sloan ${ }^{1}$, Bernhard R. Brandl ${ }^{4}$, and James R. Houck ${ }^{1}$ \\ ${ }^{1}$ Cornell University, Space Sciences Building \\ Ithaca, NY 14853-6801, USA \\ email: jbs@isc.astro.cornell.edu \\ ${ }^{2}$ University of Western Ontario, PAB 213, Canada \\ ${ }^{3}$ SETI Institute, Mountain View, CA 94043, USA \\ ${ }^{4}$ Leiden University \\ P.O. Box 9513, 2300 RA Leiden, The Netherlands
}

\begin{abstract}
One of the key questions of infrared astronomy is how the characteristics of dust depend on the physical properties of the surrounding medium. To address this question, we present results from the Spitzer Space Telescope on two projects designed to study the dust properties of a sample of 25 Planetary Nebulae (PNe) in the Magellanic Clouds, and three wellknown Giant Hiı regions (NGC 3603, 30 Doradus and N 66/NGC 346). Most PNe show emission from polycyclic aromatic hydrocarbons (PAHs) and only two of them show amorphous silicates. Eleven PNe display a strong broad feature around $11 \mu \mathrm{m}$ which is attributed to silicon carbide and 8 of them show magnesium sulfide. One PNe, SMP LMC 11, shows spectacular absorption bands due to molecules which are the precursors from which more complex hydrocarbons are formed. The Spitzer spectra of the Hil regions, NGC 3603, 30 Doradus, and NGC 346 are very rich, displaying a wealth of spectral features within each region. This not only allows us to compare the dust at different metallicities but also to study the spatial variations of many features across a given region and correlate it with the distance to the ionizing cluster(s) and other parameters.
\end{abstract}

Keywords. Infrared: general, dust, Magellanic Clouds, planetary nebulae: general, Hiı regions, ISM: individual (30 Doradus), ISM: lines and bands

\section{Introduction}

The planetary nebula phase is one of the latest phases of evolution of intermediate mass stars. Formation of dust occurs during the transition between the asymptotic giant branch (AGB) and the PN phase. Besides the strong fine-structure emission lines characteristic of PNe, the infrared spectra of PNe display a variety of dust features, which provide information on how dust is formed. In contrast, the complex structures of giant HiI regions give the opportunity to study the dust in different physical environments (distance to central cluster, shocks, etc...). In addition, it is thought that metallicity has an impact on some dust properties (Galliano et al. 2008). With this in mind, we have observed a sample of $\mathrm{PNe}$ in the Magellanic Clouds (MCs), and 3 giant Hir regions in the Milky Way (MW), LMC, and SMC using the Spitzer Space Telescope (Werner et al. 2004). For the PNe project we observed $18 \mathrm{LMC} \mathrm{PNe}$ and $7 \mathrm{PNe}$ in the SMC using the low-resolution modules (SL, LL, 5.2-37 $\mu \mathrm{m}$ ) of the Infrared Spectrograph (IRS, Houck et al. 2004). In the HII region program we used the SL module $(5.2-14 \mu \mathrm{m})$ to observed the 3 largest HiI 


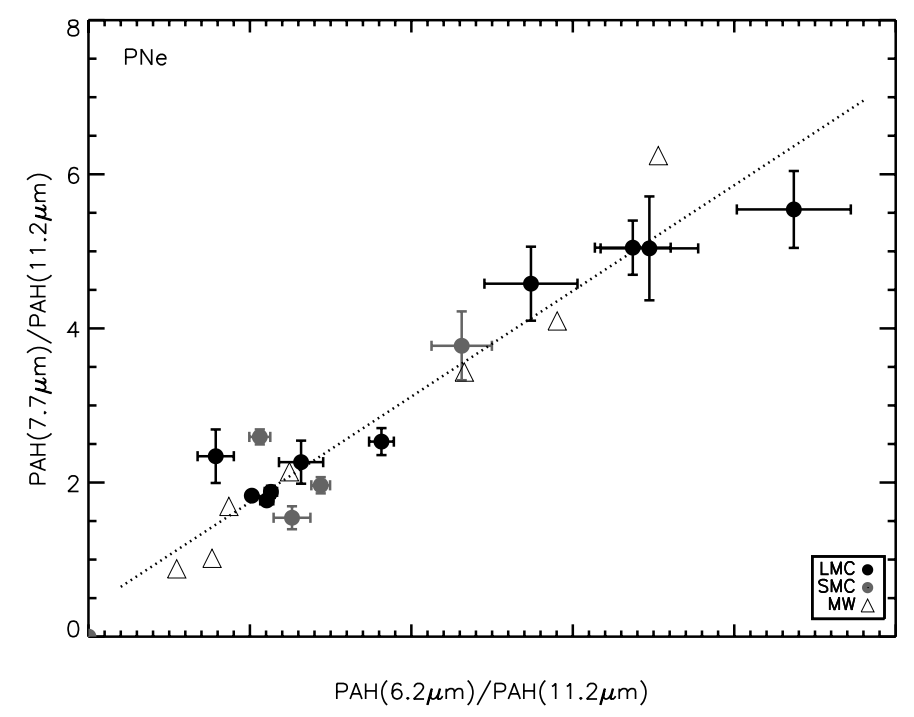

Figure 1. PAH ratio for the PNe. The dotted line is the best fit to the data.

regions in each of the following galaxies: the MW (NGC3603), the LMC (30 Doradus), and the SMC (N66).

\section{Magellanic Clouds PNe}

We have observed $25 \mathrm{PNe}$ in the MCs, 17 in the LMC and 8 in the SMC. Gas phase abundances have been derived by Bernard-Salas et al. (2008b) and the dust properties will be presented in a complementary paper (Bernard-Salas et al. 2008a). Polycyclic Aromatic Hydrocarbons (PAHs), typical of carbon-rich environments, are detected in 14 of the PNe. In addition to the 6.2, 7.7, 8.6 and $11.2 \mu \mathrm{m}$ PAH bands, in some objects with better S/N weaker bands such as the 12.7, 16.4 and $17.4 \mu \mathrm{m}$ bands are detected.

Ionized PAHs emit more in the 6.2 and $7.7 \mu \mathrm{m}$ bands while the $11.2 \mu \mathrm{m}$ band is stronger in neutral PAHs, therefore the ratio of the 6.2 or $7.7 \mu \mathrm{m}$ PAH over the $11.2 \mu \mathrm{m}$ feature can be used as a measure of the ionization fraction of the PAHs. This is shown in Figure 1 and it can be seen that there is a good correlation between these band ratios. For comparison several Galactic PNe are also plotted in Figure 1. It is interesting to note that the range of band ratios is independent of metallicity. Given that at low metallicities the hardness of the radiation field is stronger, one could have expected that the ratios of the LMC and SMC PNe would be higher (more ionised). The correlation in Figure 1 is the same of that seen in HiI regions where Galliano et al. (2008) conclude that the ionization fraction is responsible for their trend.

PAHs are known to vary from object to object and also within a class of objects. Peeters et al. (2002) proposed a classification scheme of the PAHs according to their peak position and profile. Within this classification most Galactic PNe (studied by ISO) were class B and a couple of them class A. The resolution of the SL and LL modules in the IRS is low (60-120). This makes it difficult to study variations in detail, but we can conclude that most MCs PNe are class B with a couple of them probably belonging to class A. Summarizing, the ratios of MCs PNe PAH fluxes and PAH profiles are similar to their Galactic counterparts, and independent on metallicity (at least within the metallicity range that we are probing). 


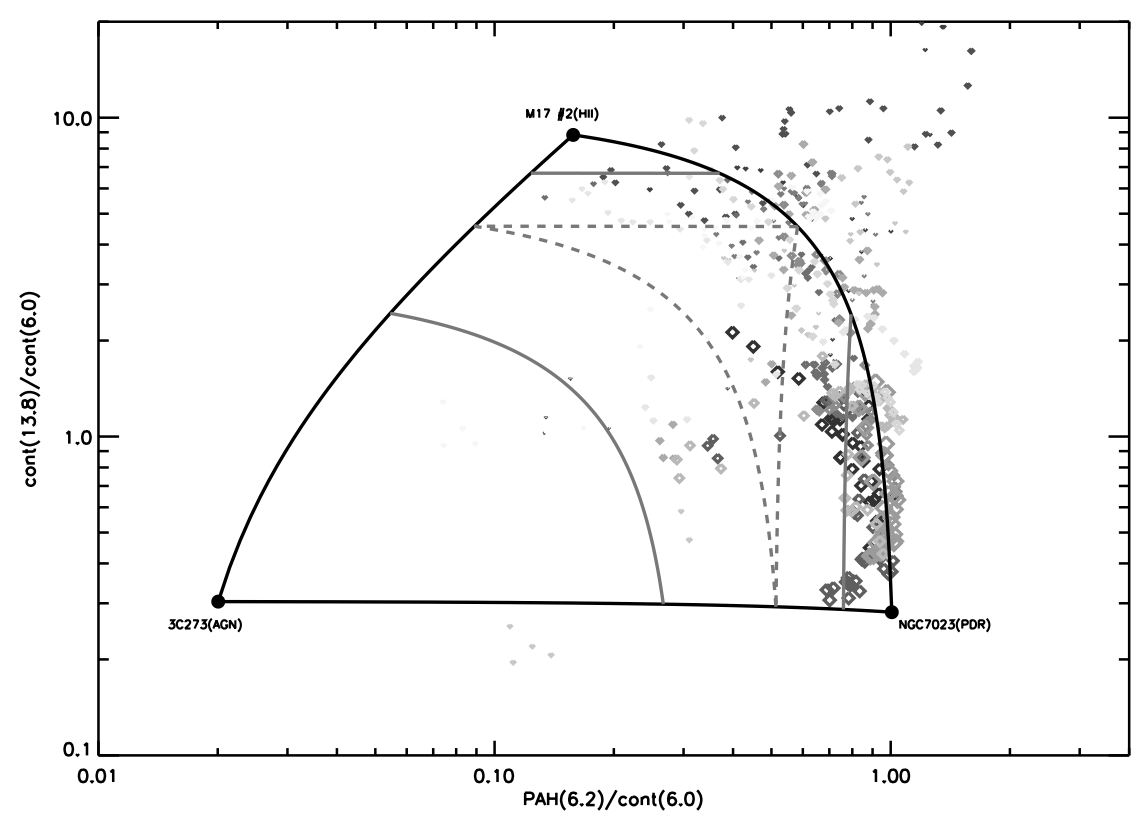

Figure 2. Laurent diagram for the different positions in 30 Doradus. The black solid lines anchor the templates for AGN, HII, and PDR dominated spectra. The grey lines indicate the contribution in percentage of each system (see Lebouteiller et al. 2007 and Bernard-Salas et al. 2008b for details).

SMP LMC 11 shows a broad absorption band in the 12-17 $\mu \mathrm{m}$ region in which narrower absorptions features are superimposed (like those of AFGL 618, Cernicharo et al. 2001), and the IR-spectrum shows that this object is in the pre-planetary nebula phase. These absorptions features correspond to acetylene, poly-acetylenic chains, benzene, and some yet un-identified features (Bernard-Salas et al. 2006), and constitute the basic molecules from which PAHs are formed. SMC SMC 24 may show hydrogenated hydrocarbons (HACs) and low excitation lines and must be a young PN. According to Sloan et al. (2007), and Sloan this volume further processing of these HACs will eventually expose the PAHs.

Of the sample of PNe showing PAHs, eight of them show silicon carbide ( $\mathrm{SiC}$ ) and eleven magnesium sulfide (MgS). These features are common of carbon-rich environments. MgS is also seen in MW PNe, but the presence of $\mathrm{SiC}$ in 8 of the $24 \mathrm{PNe}$ in the MCs (or out of 14 carbon-rich PNe) is not expected. This feature is seen in the AGB phase but it is rarely seen in Galactic PNe: only 2 MW PNe (maybe 3) show SiC. The reason for this remains unclear but it seems that the presence of this feature in the PN phase is metallicity dependent, only occurring at low metallicities. Additionally, the strength of the feature decreases with the hardness of the radiation field, either it is destroyed or processed.

Only two of the PN in the sample show amorphous silicates which is typical of oxygenrich dust. The low proportion of oxygen-rich PNe compared to the Galactic PNe is expected and it is linked to the higher efficiency of the third dredge-up (which brings out carbon) at low metallicities.

\section{Giant HiI regions}

NGC3603, 30 Doradus, and N66 are the largest Hir regions in the MW, the LMC, and SMC, respectively. Results on NGC3603 are presented in Lebouteiller et al. (2007), and 
those of 30 Doradus and N66 by Bernard-Salas et al. (2008c) and Whelan et al. (2008), respectively. A comparison of the properties of the 3 objects is given in Lebouteiller et al. (2008).

For each object several (9 to 17) positions were observed. These were centered in different locations to represent different conditions of the gas (red knots, shock fronts, stellar cluster etc.). The variety of spectra is striking, and goes from positions dominated by PAHs (e.g., red nebulosities), to fine-structure line emission with weak PAHs (e.g., close to the central cluster), to only line emission dominated spectra with no PAHs (shock front where the PAHs have been destroyed), and spectra with strong silicates absorption (embedded young star).

Taking advantage of the long slit in SL we can obtain spatial information along all the positions which were observed, this results in hundreds of spectra for each HII region. Figure 2 displays a Laurent diagram for these positions within 30 Doradus which traces very well the photo-dissociation region (PDR) and HiI region interface. The ratio of ionized to neutral PAHs shows little variation with the distance to the (main) stellar cluster in NGC3603 and 30 Doradus. This indicates that the PAH mixture is very constant over the whole region.

\section{References}

Bernard-Salas, J., Peeters, E., Sloan, G. C., et al. 2006, ApJL, 652, 29

Bernard-Salas, J., Peeters, E., Sloan, G. C., et al. 2008a, in prep

Bernard-Salas, J., Pottasch, S. R., Gutenkunst, S., et al. 2008b, ApJ, 672, 274

Bernard-Salas, J., et al. 2008c, in prep

Cernicharo, J., Heras, A. M., Tielens, A. G. G. M., et al. 2001, ApJ, 546, L123

Galliano, F., Madden, S. C., Tielens, A. G. G. M., Peeters, E., \& Jones, A. P. 2008, ApJ, in press

Houck, J. R., Roellig, T. L., van Cleve, J., et al., 2004, ApJS, 154, 18

Laurent, O., Mirable, I. F., Charmandaris, V., et al. 2000, $A \mathscr{G} A, 359,887$

Lebouteiller, V., et al. 2007, ApJ, 655, 390

Lebouteiller, V., et al. 2008, ApJ, in prep

Peeters, E., Van Kerckhoven, C., Tielens, A. G. G. M., et al. 2002, A\&A, 390, 1089

Sloan, G. C., Duley, W. W., Kraemer, K. E., et al. 2007, ApJ, 664, 1144

Werner, M. W., Roellig, T. L., Low, F. J., et al., 2004, ApJS, 154, 1

Whelan, D., et al. 2008, in prep

\section{Discussion}

Mulas: A comment about the band ratios that you use to determine ionization of PAHs; while is it true that ionization has a large effect, it is not the only parameter. It is a multidimensional space. Especially the ratio of the $11.3 \mu \mathrm{m}$ PAH band with shorter wavelength bands. The $11.3 \mu \mathrm{m}$ band is not so weak in ionized big PAHs, so you should be a bit careful with the interpretation.

BERNARD-SALAS: Indeed, there are other processes besides ionization that could a priori explain the correlation we see in the PAH ratios, the main ones being dehydrogenation, extinction and to a lesser extent molecular size and structure. Dehydrogenation is only relevant for molecules with less than $25 \mathrm{C}$-atoms, and the PAHs we believe are present in space are larger than that. We can rule out extinction due to silicates which affects the $11.3 \mu \mathrm{m}$ band because these PNe have no silicates. Additionally, in the 30 Dor spectra the positions with silicate absorption and the ones without it show the same trend. Also, in a recent study by Galliano et al. (2008) they study the same PAH ratio for a great variety of sources. Their ratios differ by a factor of 10 and they conclude that only ionization can 
produce such a large effect. The PNe we present here have ratios differing by a factor of 3 but they fall right in the trend seen by Galliano and collaborators, so while we cannot rule out other options it is most likely that ionization drives the relation that is shown. Concerning your last comment, Bauschlicher et al. (2008) found that PAH cations in large PAHs do not have a major contribution to the $11.3 \mu \mathrm{m}$ band, but I will be very interested if this is not the case.

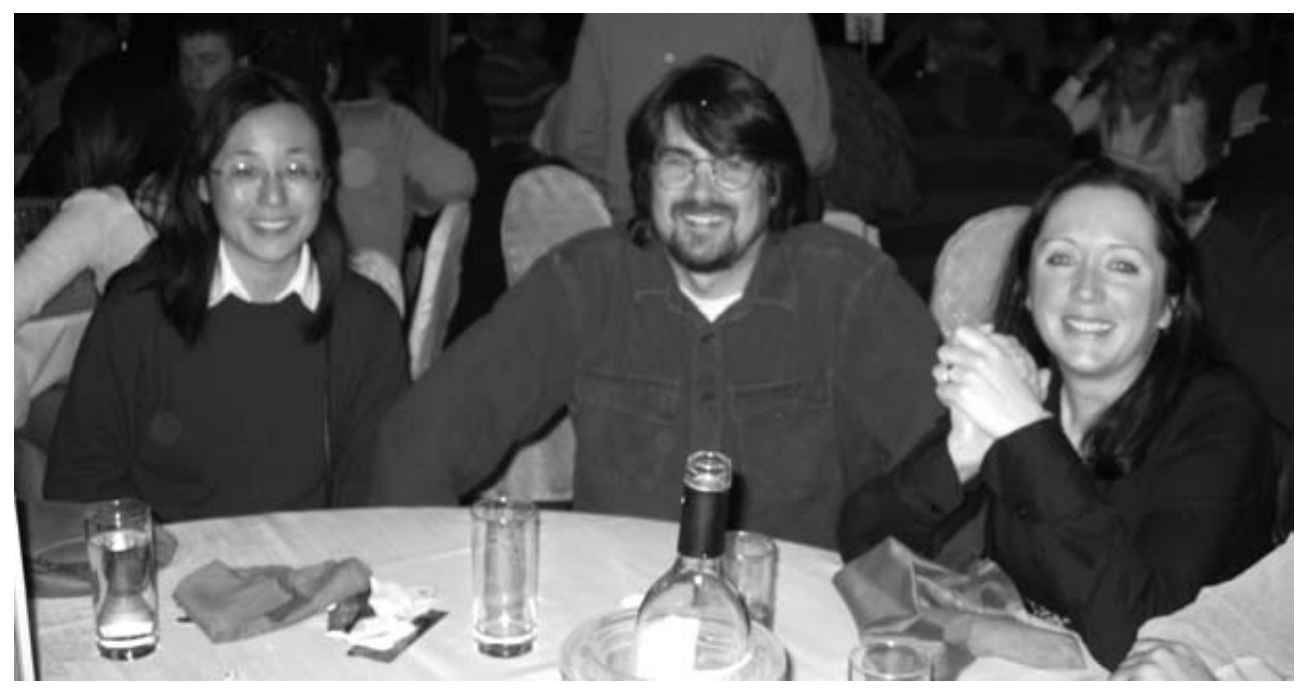

From left to right: Mikako Matsurra, Greg Sloan, Angela Speck 


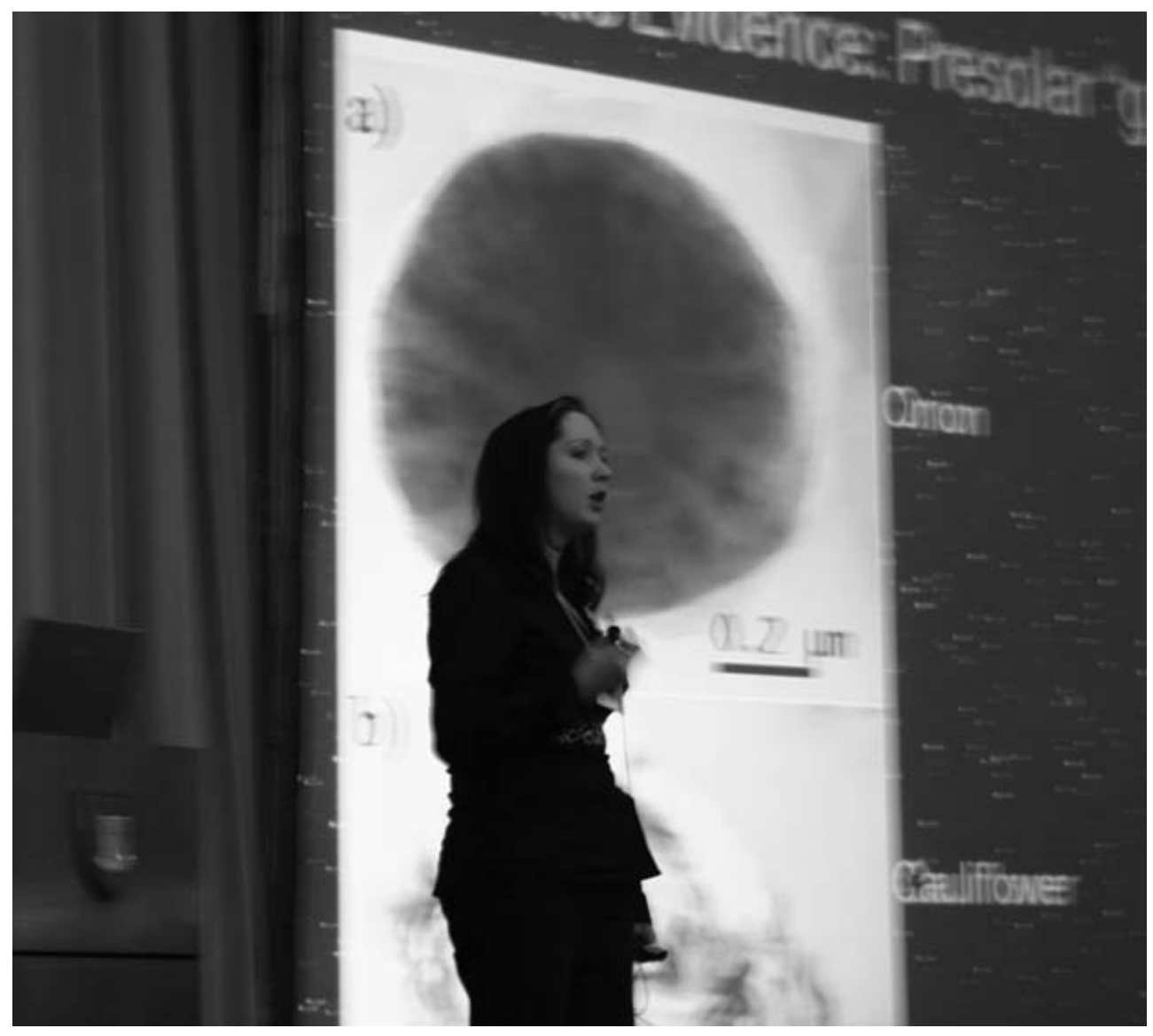

Angela Speck talking about pre-solar grains. 